\title{
Характеристики барьеров Шоттки тонкопленочных, двухконтактных структур Al/пленка из наночастиц Si/ITO
}

\author{
(C) Н.Н. Кононов ${ }^{1}$, С.Г. Дорофреев ${ }^{2}$ \\ ${ }^{1}$ Институт общей физики им. А.М. Прохорова Российской академии наук, \\ 119991 Москва, Россия \\ ${ }^{2}$ Московский государственный университет им. М.И. Ломоносова (химический фракультет), \\ 119991 Москва, Россия \\ E-mail: nnk@kapella.gpi.ru
}

(Получена 18 октября 2016 г. Принята к печати 24 октября 2016 г.)

Температурная зависимость высот барьеров Шоттки и последовательного сопротивления тонкопленочных структур Al/пленка из наночастиц $\mathrm{Si} / \mathrm{ITO}$ (Al/nc-Si film/ITO) определены из анализа вольт-амперных характеристик в диапазоне температур $20-150^{\circ} \mathrm{C}$. Обнаружено, что вид вольт-амперной характеристики при всех исследованных температурах может быть описан моделью двух диодов Шоттки, включенных навстречу друг другу. Для двух диодов Шоттки, включенных навстречу друг другу, получена общая формула, позволяющая конструировать функции, аппроксимирующие экспериментальные кривые с высокой точностью. На основе этой формулы построена вычислительная модель, обобщающая теоретические результаты работы C.K. Ланга и H.B. Чанга (S.K. Cheung and N.W. Cheung), широко применяемые для анализа вольт-амперных характеристик одиночных диодов Шоттки. В результате нами разработана методика, позволяющая вычислять высоты барьеров Шоттки в системе двух диодов, включенных навстречу друг другу, коэффициенты их неидеальности и последовательное сопротивление системы. Обнаружено, что в исследованном температурном интервале величины высот барьеров находятся вблизи значений $\sim 1$ эВ. Из анализа температурной зависимости высот барьеров установлено, что столь большие величины связаны с наличием на границах наночастиц кремния окисного слоя $\mathrm{SiO}_{x}(0 \leq x \leq 2)$, преодолевать который носители заряда могут как в результате теплового возбуждения, так и в результате туннелирования. Установлено, что собственные высоты барьеров Шоттки переходов $\mathrm{Al} / n c$-Si film и $n c$-Si film/ITO составляют $\sim 0.1$ эВ. Из анализа активационных зависимостей для последовательного сопротивления структур $\mathrm{Al} / n c-\mathrm{Si}$ film/ITO и из импеданс-спектров выяснено, что в структурах реализуется комбинированный механизм транспорта электрических зарядов, связанный с ионной и электронной проводимостью. Установлено, что с ростом температуры образца вклад электронной проводимости в суммарный процесс транспортировки зарядов увеличивается.

DOI: $10.21883 /$ FTP.2017.05.44421.8435

\section{1. Введение}

Полупроводниковые наночастицы в последние годы вызывают у исследователей большой интерес, обнаруживая много уникальных оптических и электрических свойств.

Электронные приборы на основе тонких пленок из полупроводниковых наночастиц обязательно контактируют с внешним электрическим контуром, поэтому электронные свойства переходов пленка/подводящий электрод играют весьма заметную роль в характеристиках таких приборов. В частности, барьеры Шоттки, которые могут формироваться на границе полупроводниковая пленка/металлический электрод, существенно изменяют электрические свойства подобных тонкопленочных структур [1].

В настоящее время чрезвычайно большие перспективы имеют тонкопленочные наноструктуры металл/полупроводниковая пленка/металл, поскольку такие структуры открывают большие возможности для создания гибких электронных приборов, таких, например, как элементы памяти [2], тонкопленочные транзисторы $[3,4]$ и газовые сенсоры [5].
Кроме того, электрические параметры, характерные для таких двухтерминальных структур, обнаруживаются в миниатюрных полупроводниковых приборах на основе одиночных нанообъектов (single nano-objects), например на основе нанонитей (single nanowire devices): биосенсоры [6] и тонкопленочные полевые транзисторы (nanowire field effect transistors) [7,8].

Так как параметры подобных приборов в значительной мере определяются свойствами перехода полупроводник/подводящий контакт, анализ изменения транспортных параметров таких двухтерминальных структур с температурой, частотой и величиной приложенного внешнего напряжения имеет большое практическое значение.

Важной особенностью электрических характеристик структур металл/полупроводник/металл во многих случаях является формирование в них двух диодов Шоттки, включенных навстречу друг другу [9-11]. Существует большое число публикаций, в которых приводится анализ подобных характеристик [12-14], из которого определяются высота барьера Шоттки, коэффициент неидеальности и другие параметры таких структур. В этой статье мы представляем результаты измере- 
ний и анализа вольт-амперных характеристик (BAX) и импеданс-спектров тонкопленочных структур, состоящих из тонких пленок наночастиц $\mathrm{Si}(n c-\mathrm{Si}$ film), нанесенных на прозрачную подложку, покрытую проводящим электродом из оксида индия-олова (ITO) и последующего напыления на них верхнего алюминиевого электрода (Al/nc-Si film/ITO). Подобная структура с прозрачным ITO электродом имеет большие перспективы для применения в различных оптоэлектронных приборах и солнечных батареях.

BAX этих структур измерялись в диапазоне температур $20-150^{\circ} \mathrm{C}$. Мы предлагаем расчетную формулу, которая применима для вычисления высот барьеров и коэффициентов неидеальности в структурах с двумя диодами Шоттки с различной высотой, включенными навстречу друг другу. Эта формула также позволяет вычислять последовательное сопротивление, которым может обладать такая система.

Мы также приводим расчетную методику, которая обобщает теоретическое рассмотрение, проведенное S.K. Cheung и N.W. Cheung [15] для прямой вольт-амперной характеристики одиночного диода Шоттки. Сравнение величин коэффициентов неидеальности структур Al/nc-Si film/ITO, вычисленных по методу [15] и по нашей методике, показывает, что в первом случае получаются неправдоподобно большие значения, в то время как значения коэффициентов, вычисленных по предлагаемой нами методике, оказываются весьма близкими к единице.

\section{2. Детали эксперимента}

Для формирования пленок из нанокристаллического кремния использовались кремниевые наночастицы, полученные в результате лазерно-индуцированного пиролиза силана [16]. Средний диаметр наночастиц кремния (nc-Si), полученных пиролизом силана, составлял 12 нм, их внешние оболочки были частично окислены и представляли собой $\mathrm{SiO}_{x}(0 \leq x \leq 2)$. Удельная поверхность наночастиц, измеренная методом адсорбции азота (ВЕТ method), составляла $220 \mathrm{~m}^{2} /$ г. Для приготовления слоистых структур на стеклянные подложки с покрытием из оксида индия-олова посредством электрофореза или высоковольтного электрораспыления из золей, содержащих наночастицы кремния $(n c-\mathrm{Si})$, наносились пленки $n c-\mathrm{Si}$, толщина которых изменялась в диапазоне от $50 \mathrm{Hм}$ до 1 мкм. Толщина пленок измерялась профилометром TalyStep (Taylor-Hobbson). Затем на полученную слоистую структуру $n c-\mathrm{Si}$ film/ITO в вакууме напылялся верхний металлический электрод из алюминия. Получившуюся тонкопленочную структуру в дальнейшем мы будем обозначать Al/nc-Si film/ITO. Геометрия изученных образцов показана на рис. 1.

Для измерения вольт-амперных характеристик использовались стенды, которые позволяли регистрировать электрические параметры образцов в диапазоне

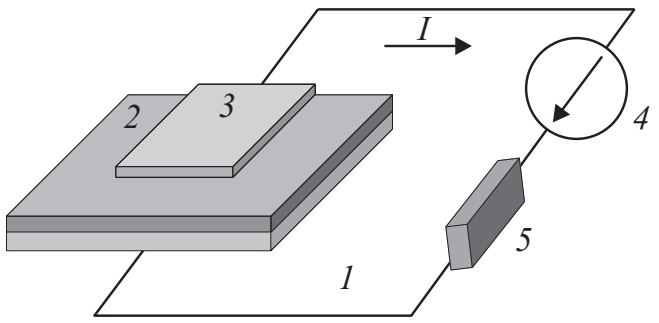

Рис. 1. Геометрия слоистых тонкопленочных структур $\mathrm{Al} / n c$-Si film/ITO. 1 - стеклянная подложка с покрытием ITO, 2 - пленка $n c-\mathrm{Si}, 3$ - верхний алюминиевый электрод, 4 - источник внешнего напряжения, 5 - сопротивление нагрузки. Стрелкой показано направление тока, соответствующее положительному напряжению на структуре.

температур $20-150^{\circ} \mathrm{C}$ с точностью $0.1^{\circ} \mathrm{C}$. В качестве регистрирующих приборов использовались высокоомные 24-битные 4-канальные цифровые самописцы Эксперт-001 (Эконикс). В проведенной серии измерений напряжение считалось положительным, когда потенциал нижнего электрода был больше потенциала верхнего.

Также проводились измерения импедансов структур $\mathrm{Al} / n c-\mathrm{Si}$ film/ITO в указанном интервале температур и в области частот $1-10^{6}$ Гц с помощью импедансметра „Элинс 3000“.

\section{3. Результаты и обсуждение}

\section{1. Экспериментальные BAX структур Al/nc-Si film/ITO}

ВАХ исследованных элементов во всем диапазоне температур 20- $150^{\circ} \mathrm{C}$ имеет циклическую структуру, подобную структуре кривых, приведенных на рис. 2. Такая структура характерна для элементов, обладающих некоторой емкостью (electrochemical capacity), которая

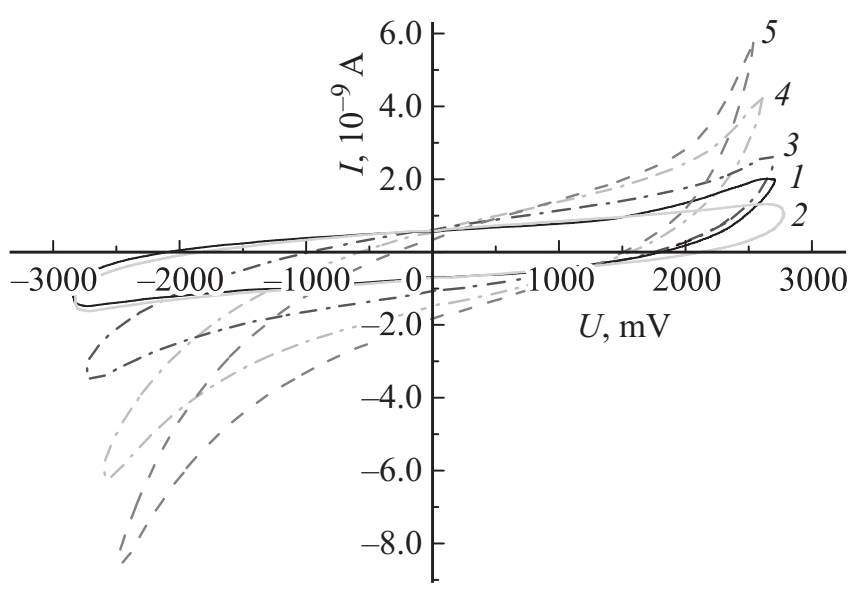

Рис. 2. Циклические $\mathrm{BAX}$ элемента $\mathrm{Al} / n c-\mathrm{Si}$ film/ITO, измеренные при разных температурах, ${ }^{\circ} \mathrm{C}: 1-22,2-61$, $3-100,4-119,5-140$. 
связана с поляризацией двойного слоя, существующего на границе полупроводник/электролит. Для пленки $n c-\mathrm{Si}$ подобная ситуация связана с присутствием молекул воды вблизи поверхности наночастиц кремния.

Дифференциальная электрохимическая емкость может быть определена из соотношения: $C=I(\Delta V / \Delta t)^{-1}$, где $\Delta V / \Delta t-$ скорость изменения внешнего напряжения, прикладываемого к образцу. Мы регистрировали $I(V)$ при $\Delta V / \Delta t=10^{-3} \mathrm{~B} / \mathrm{c}$. В рамках данной статьи мы не будем анализировать причины, приводящие к появлению электрохимической емкости элемента $\mathrm{Al} / n c$-Si film/ITO и ее параметры. Отметим только, что при анализе экспериментальных кривых в аппроксимирующие функции вводилось постоянное смещение, связанное с вкладом в суммарный ток, ток зарядки соответствующей емкости. Также отметим, что в исследованных образцах мы обнаружили спонтанную генерацию эдс. Причины генерации эдс и ее параметры будут рассмотрены в другой статье. Здесь же отметим, что наличие эдс образца также учитывалось при построении аппроксимирующих функций.

\section{2. Методика вычисления параметров диодов Шоттки, включенных навстречу друг другу}

Во всем диапазоне регистрируемых температур ВАХ структур Al/nc-Si film/ITO имеют вид, характерный для двух диодов Шоттки, включенных навстречу друг другу (см. рис. 2).

Как уже отмечалось, анализ подобных ВАХ проведен в довольно большом количестве публикаций (см., например, [12-14]). Мы в данной статье приводим полученную нами формулу, которая позволяет аппроксимировать экспериментальные кривые с высокой точностью и позволяет определять высоты барьеров диодов Шоттки, общее последовательное сопротивление и коэффициенты их неидеальности. Первое утверждение, которое мы используем, заключается в том, что для системы диодов Шоттки, включенных навстречу друг другу так, как показано на рис. 3, падение напряжения $V_{1}$ на диоде, включенном в прямом направлении, меньше напряжения $V_{2}$ на диоде, включенном навстречу, даже если барьеры одинаковые. Действительно, ширины обедненных слоев $d_{1}$ и $d_{2}$ в полупроводнике вблизи барьеров определяются формулой [17]:

$$
d_{1,2}=\left(\frac{2 \varepsilon \varepsilon_{0}\left(\Phi_{0}-k T \mp e V_{1,2}\right)}{e^{2} n_{0}}\right)^{1 / 2} .
$$

Здесь $\Phi_{0}=\Phi_{01}=\Phi_{02}$ - высота барьеров со стороны полупроводника, $\Phi_{0}=e V_{0}$, где $V_{0}$ - встроенный потенциал, $n_{0}$ - равновесная концентрация зарядов в полупроводнике, $\varepsilon_{0}, \varepsilon-$ диэлектрическая постоянная вакуума и проницаемость полупроводника, $e=1.6 \cdot 10^{-19}$ Кл элементарный заряд. Из приведенной формулы следует, что $d_{2}>d_{1}$, и если полагать, что напряженность внешнего электрического поля в полупроводнике однородна, то $V_{1} \sim d_{1}$ и $V_{2} \sim d_{2}$, т. е. $V_{2}>V_{1}$.

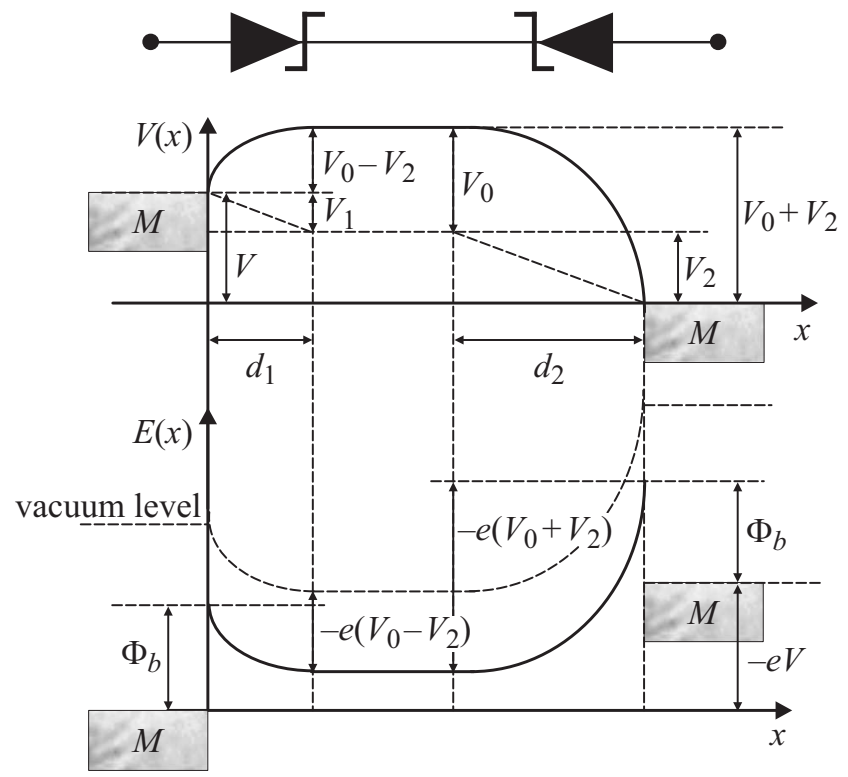

Рис. 3. Схема распределения потенциалов и энергий в структуре двух одинаковых диодов Шоттки, включенных навстречу друг другу (сопротивление полупроводника на схеме не учитывается). Потенциал левого электрода больше потенциала правого.

На рис. 3 приведена схема распределения потенциалов и энергий в системе двух встречных одинаковых диодов Шоттки без учета последовательного сопротивления полупроводника, при приложении к системе разности потенциалов $V$ так, что левый потенциал больше правого. Эта схема иллюстрирует утверждения $d_{2}>d_{1}$ и $V_{2}>V_{1}$.

С учетом неидеальности диодов ток через них описывается следующими уравнениями [12]:

$$
\begin{gathered}
I_{1}=I_{01} e^{e V_{1} / n k T}\left(1-e^{-e V_{1} / k T}\right) ; \\
I_{2}=-I_{02} e^{-e V_{2} / n k T}\left(1-e^{e V_{2} / k T}\right) .
\end{gathered}
$$

В этих уравнениях $n-$ коэффициент неидеальности диода, связанный с зависимостью высоты барьера от эффекта Шоттки и от туннелирования зарядов под барьером. В представленной модели полагается, что коэффициенты неидеальности одинаковые для обоих диодов. В приближении теории термоэлектронной эмиссии токи насыщения $I_{01}$ и $I_{02}$ определяются соотношением

$$
I_{01,2}=S A^{*} T^{2} e^{-\Phi_{b 1,2} / k T},
$$

где $\Phi_{b 1}$ и $\Phi_{b 2}$ - высота барьеров со стороны металла (см. рис. 3), $A^{*}$ - эффективная постоянная Ричардсона. В случае использования электродов из одного металла и в отсутствие поверхностных состояний на границах металл/полупроводник эти величины должны быть одинаковыми для обоих электродов. Если ввести эффективную высоту барьеров $\Phi_{b 1}^{\mathrm{eff}}$ и $\Phi_{b 2}^{\mathrm{eff}}$ : 
$\Phi_{b 1,2}^{\mathrm{eff}}\left(V_{1,2}\right)=\Phi_{b 1,2} \pm e V_{1,2}(1-1 / n), \quad$ то уравнения (2) примут вид, характерный для идеальных диодов Шоттки:

$$
I_{1}=I_{01}^{\mathrm{eff}}\left(e^{e V_{1} / k T}-1\right) ; \quad I_{2}=-I_{02}^{\mathrm{eff}}\left(e^{-e V_{2} / k T}-1\right),
$$

где

$$
I_{01,2}^{\mathrm{eff}}=S A^{*} T^{2} e^{\frac{-\Phi_{b_{1,2}}^{\mathrm{eff}}\left(V_{1,2}\right)}{k T}} .
$$

Так как диоды включены последовательно, то $V_{1}+V_{2}=V, I_{1}=I_{2}=I$, где $V$ - общее напряжение, приложенное к системе диодов, и $I-$ ток текущий через нее. Падение напряжения на диодах $V_{1}$ и $V_{2}$ можно связать с общим напряжением $V$, если положить $V_{1}=\alpha V$ и $V_{2}=(1-\alpha) V$. В этом случае для силы тока, текущего через такую систему диодов, справедливо следующее соотношение (вывод см. в Приложсении):

$$
I=\frac{I_{01} I_{02}\left[e^{\frac{e V_{2}}{k T}}-e^{\frac{-e V_{1}}{k T}}\right]}{\left[I_{01} e^{\frac{e V_{2}}{n k T}}+I_{02} e^{\frac{-e V_{1}}{n k T}}\right]}=\frac{I_{01}\left[e^{\frac{e(1-\alpha) V}{k T}}-e^{\frac{-e \alpha V}{k T}}\right]}{\left[\frac{I_{01}}{I_{02}} e^{\frac{e(1-\alpha) V}{n k T}}+e^{\frac{-e \alpha V}{n k T}}\right]} .
$$

Величины $I_{01}, I_{02}, \alpha$ и $n$, в формуле (5) являются подгоночными параметрами и определяются в процессе аппроксимации данным соотношением экспериментальной кривой. Формула (5) не учитывает последовательного сопротивления $R$, которым может обладать исследуемая система. С учетом последовательного сопротивления уравнение (5) можно записать в виде:

$$
\begin{aligned}
I & =\frac{I_{01} I_{02}\left[e^{\frac{e\left(V_{2}-I R\right)}{k T}}-e^{\frac{-e\left(V_{1}-I R\right)}{k T}}\right]}{\left[I_{01} e^{\frac{e\left(V_{2}-I R\right)}{n k T}}+I_{02} e^{\frac{-e\left(V_{1}-I R\right)}{n k T}}\right]} \\
& =\frac{I_{01}\left[e^{\frac{e[(1-\alpha) V-I R]}{k T}}-e^{\left.\frac{-e[\alpha V-I R]}{k T}\right]}\right.}{\left[\frac{I_{01}}{I_{02}} e^{\frac{e[(1-\alpha) V-I R]}{n k T}}+e^{\frac{-e[\alpha V-I R]}{n k T}}\right]} .
\end{aligned}
$$

$R$ также является подгоночным параметром, и так как всего таких параметров 5, то существует неоднозначность в их выборе. Поэтому для определения $R$ мы модифицировали методику, разработанную S.K. Cheung и N.W. Cheung [15].

Суть нашего метода заключается в следующем, при $V \geq 3 k T$ формула (5а) записывается в виде

$$
I \approx I_{02} e^{\frac{e\left(V_{2}-I R\right)\left(1-\frac{1}{n}\right)}{k T}}
$$

Это соотношение отражает тот простой факт, что при большом напряжении сила тока, текущего через систему диодов определяется диодом, включенным во встречном направлении. Соответственно

$$
V_{2}=I R+\frac{k T}{e\left(1-\frac{1}{n}\right)} \ln \left(\frac{I}{I_{02}}\right) .
$$

Здесь $I_{02}$ - ток насыщения, определяемый формулой (3), поэтому уравнение (7) можно записать в виде

$$
\begin{aligned}
V_{2} & =I R+\frac{k T}{e\left(1-\frac{1}{n}\right)} \ln \left(\frac{I}{\left(S A^{*} T^{2}\right.} e^{\frac{\Phi_{b 2}}{k T}}\right) \\
& =I R+\frac{k T}{e\left(1-\frac{1}{n}\right)} \ln \left(\frac{I}{S A^{*} T^{2}}\right)+\frac{1}{e\left(1-\frac{1}{n}\right)} \Phi_{b 2} .
\end{aligned}
$$

Продифференцировав уравнение (8) по $\ln \left(I^{*}\right)$, где $I^{*}=I / S A^{*} T^{2}$, получим

$$
\frac{d V_{2}}{d\left(\ln I^{*}\right)}=I R+\frac{k T}{e(1-1 / n)} .
$$

Уравнение (9) по виду похоже на уравнение для $d V / d(\ln I)$, полученное в работе [15], за исключением того, что в качестве напряжения необходимо брать разность потенциалов на диоде, включенном навстречу. Еще одно важное отличие заключается в том, что в свободном члене этого уравнения вместо величины $\frac{k T}{e} n$ стоит выражение $\frac{k T}{e}\left(1-\frac{1}{n}\right)^{-1}$. Поэтому график функции $d\left(V_{2}\right) / d\left(\ln \left(I^{*}\right)\right)$ в зависимости от $I$, который, как видно из (9), представляет собой прямую линию, будет пересекать ось абсцисс в точке $\frac{k T}{e}\left(1-\frac{1}{n}\right)^{-1}$, а не в точке $\frac{k T}{e} n$. Это, на наш взгляд, очень важное отличие, так как если пользоваться методикой работы [15], то должны получаться большие значения коэффициента $n(\sim 10)$, что противоречит самому определению этого параметра, как малому отклонению от идеальности диода. Также из наклона прямой $d\left(V_{2}\right) / d\left(\ln \left(\left(I^{*}\right)\right)\right.$ находится величина $R$ последовательного сопротивления системы диодов.

Аналогично работе [15] введем функцию

$$
H(I) \equiv V_{2}-\frac{k T}{e\left(1-\frac{1}{n}\right)} \ln \left(\frac{I}{\left(S A^{*} T^{2}\right.}\right),
$$

тогда в соответствии с уравнением (8) получим следующее соотношение:

$$
H(I)=I R+\frac{1}{e\left(1-\frac{1}{n}\right)} \Phi_{b 2} .
$$

Из этого соотношения видно, что $H$ линейно зависит от силы тока и величина $R$ определяется как наклон прямой $H(I)$. Точка пересечения $H(I)$ с осью абсцисс дает величину барьера $\Phi_{b 2}$.

Теперь опишем методику, применяемую нами для аппроксимации экспериментальных ВАХ. Эта методика описывается следующими этапами.

1) экспериментальная ВАХ аппроксимируется зависимостью (5) в предположении, что $R=0$. Из этой аппроксимации в первом приближении находятся $n_{1}$ и соотношение $\left(V_{2} / V_{1}\right)_{1}$ и в соответствии с $V$ значение $V_{2}$;

2) на основании экспериментальных данных строится функция $d\left(V_{2}\right) / d\left(\ln \left(I_{\exp }^{*}\right)\right)$ и из графика $d\left(V_{2}\right) / d\left(\ln \left(I_{\exp }^{*}\right)\right)$ 


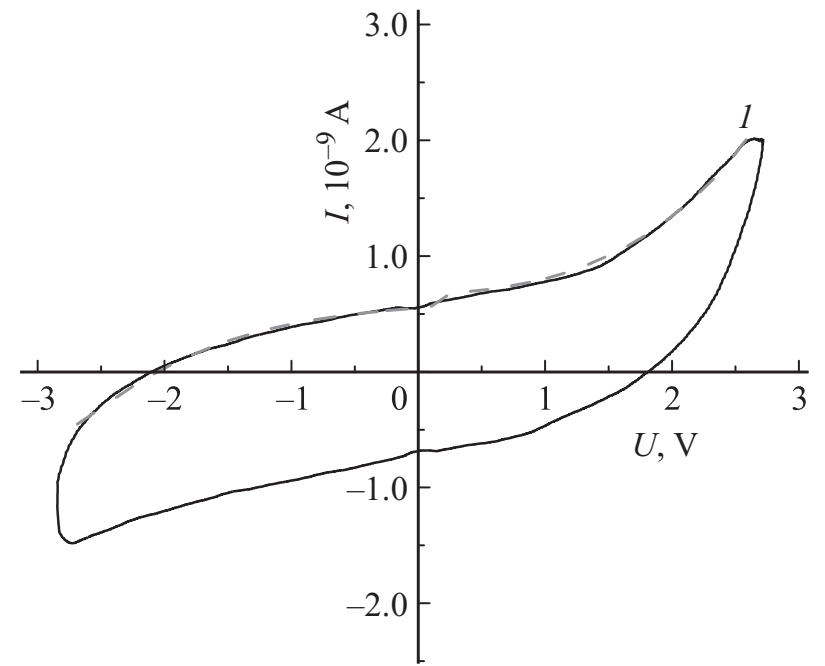

Рис. 4. $\mathrm{BAX}$ элемента $\mathrm{Al} / n c$-Si film/ITO, измеренная при температуре $22^{\circ} \mathrm{C}$, пунктирной линией показана аппроксимация в соответствии с методикой, описанной в этой статье.

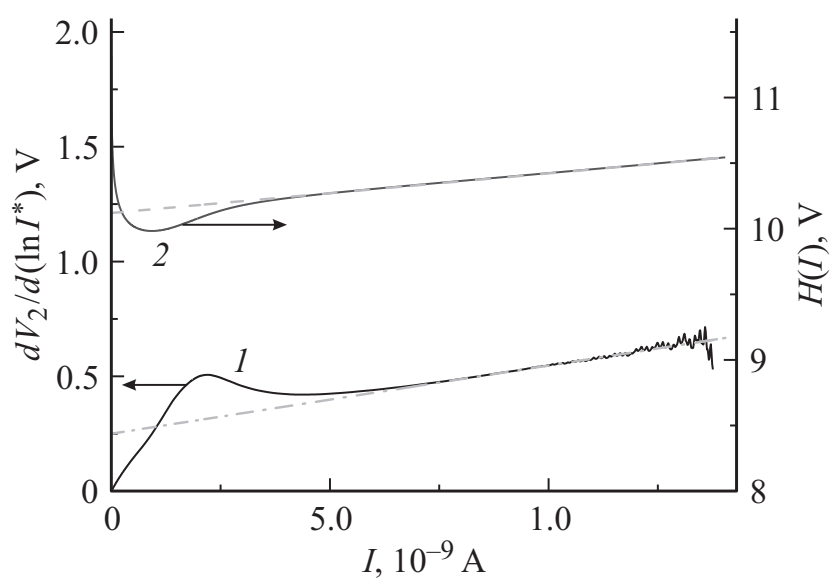

Рис. 5. Графики зависимости функций $d V_{2} / d\left(\ln I^{*}\right) \quad(1)$ $H(I)$ (2) (объяснение см. в тексте) от тока, текущего через элемент $\mathrm{Al} / n c$-Si film/ITO, находящийся при температуре $22^{\circ} \mathrm{C}$.

в зависимости от $I_{\exp }$ определяются величины $n$ и $R$. Отметим, что значение $n$ определяется независимо и в принципе может отличаться от $n_{1}$. Если значения величин $n_{1}$ и $n$ близки, то это указывает на достаточную точность подгонки экспериментальной кривой с помощью уравнения (5);

$3)$ значения $n$ и $R$, с учетом первичной аппроксимации, подставляются в уравнение (5а) и во втором приближении определяется скорректированное соотношение $\left(V_{2} / V_{1}\right)_{2}$;

4) повторяется процедура 2), в результате которой определяются исправленные значения $n$ и $R$;

$5)$ вводится функция $H(I)$ и из графика $H(I)$ в зависимости от $I_{\exp }$ определяются $\Phi_{b 2}$ и еще одно значение $R$.

На рис. 4 приведена экспериментальная ВАХ структуры Al/nc-Si film/ITO, измеренная при температуре $22^{\circ} \mathrm{C}$, и ее аппроксимация, проведенная по описанной выше методике. На этом рисунке приведена только аппроксимация верхней части циклической $\mathrm{BAX}$, поскольку аппроксимация ее нижней части проводится совершенно аналогично. Сопоставление экспериментальных ВАХ, измеренных при других температурах образца и соответствующих аппроксимирующим функциям, демонстрирует высокую точность изложенного метода.

Функции $d\left(V_{2}\right) / d\left(\ln \left(I_{\text {exp }}^{*}\right)\right)$ и $H$ в зависимости от силы тока, протекающего через элемент $\mathrm{Al} / n c-\mathrm{Si}$ film/ITO и соответствующего рис. 4, приведены на рис. 5. Из этого рисунка видно, что функции $d\left(V_{2}\right) / d\left(\ln \left(I_{\exp }^{*}\right)\right)$ и $H$ линейны только в области достаточно больших токов, соответственно эти области и служили для определе-

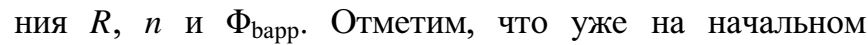
этапе подгонки величины $n_{1}$ и $n$ достаточно близки, так функция, аппроксимирующая BAX, на рис. 3 построена с учетом $n=1.08$, в то время как $n_{1}$, определенная из зависимости $d\left(V_{2}\right) / d\left(\ln \left(I_{\exp }^{*}\right)\right)$, составляет 1.1 .

\section{3. Вычисление высот барьеров с учетом туннелирования через слой диэлектрика, разделяющего металл-полупроводник}

Из анализа аппроксимирующих функций следует, что высоты барьеров Шоттки $\Phi_{b 1,2}$ линейно увеличиваются с ростом абсолютной температуры образца (см. рис. $6, a$ ). Такое поведение возникает в том случае, когда металл и полупроводник, образующие барьер Шоттки, разделены слоем диэлектрика и носители заряда, пересекающие границу, туннелируют через этот слой. Как показали Card и Rhoderick [18], в случае диода Шоттки с таким интерфейсным слоем прямой ток насыщения $(V \geq 3 k T)$ определяется выражением:

$$
I_{0}^{\mathrm{app}}=S A^{*} T^{2} e^{-\alpha \chi^{0.5} \delta} e^{\frac{-\Phi_{b}(0)}{k T}} .
$$

Здесь $\Phi_{b}(0)$ - собственная высота барьера, $\chi$ (в эВ) средняя высота барьера и $\delta$ (в $\AA)$ - толщина интерфейсного слоя. Постоянная $\alpha=\left[2\left(2 m^{*} / \hbar^{2}\right)\right]^{1 / 2}$, где $m^{*}$ - эффективная масса носителей, примерно равна 1 эВ $\mathrm{B}^{-1 / 2} \AA^{-1}$ и может быть опущена. Член $\alpha \chi^{1 / 2} \delta$ называется туннельным фактором. Соответственно

$$
\Phi_{b}^{\mathrm{app}}(T)=\Phi_{b}(0)+k T \alpha \chi^{1 / 2} \delta .
$$

Из уравнения (10) видно, что $\Phi_{b}^{\text {app }}$ линейно зависит от температуры, причем наклон функции $\Phi_{b}^{\text {app }}(T)$ дает величину туннельного фактора, а пересечение с осью абсцисс $(T=0)$ величину $\Phi_{b}(0)$. Из линейных аппроксимаций экспериментальных зависимостей $\Phi_{b 1,2}(T)$, приведенных на рис. $6, a$, следует, что $\Phi_{b 1}(0)=0.115$ эВ, $\Phi_{b 2}(0)=0.095$ эВ и фактор туннелирования для этих барьеров составляет 34.78 и 36.0.

Если воспользоваться результатами работы [18], то значения $\chi^{1 / 2} \delta>10$ соответствуют толщинам интерфейсного слоя $\mathrm{SiO}_{2}$, превосходящим $\sim 1$ нм. Этот результат хорошо коррелирует с нашими более ранними 

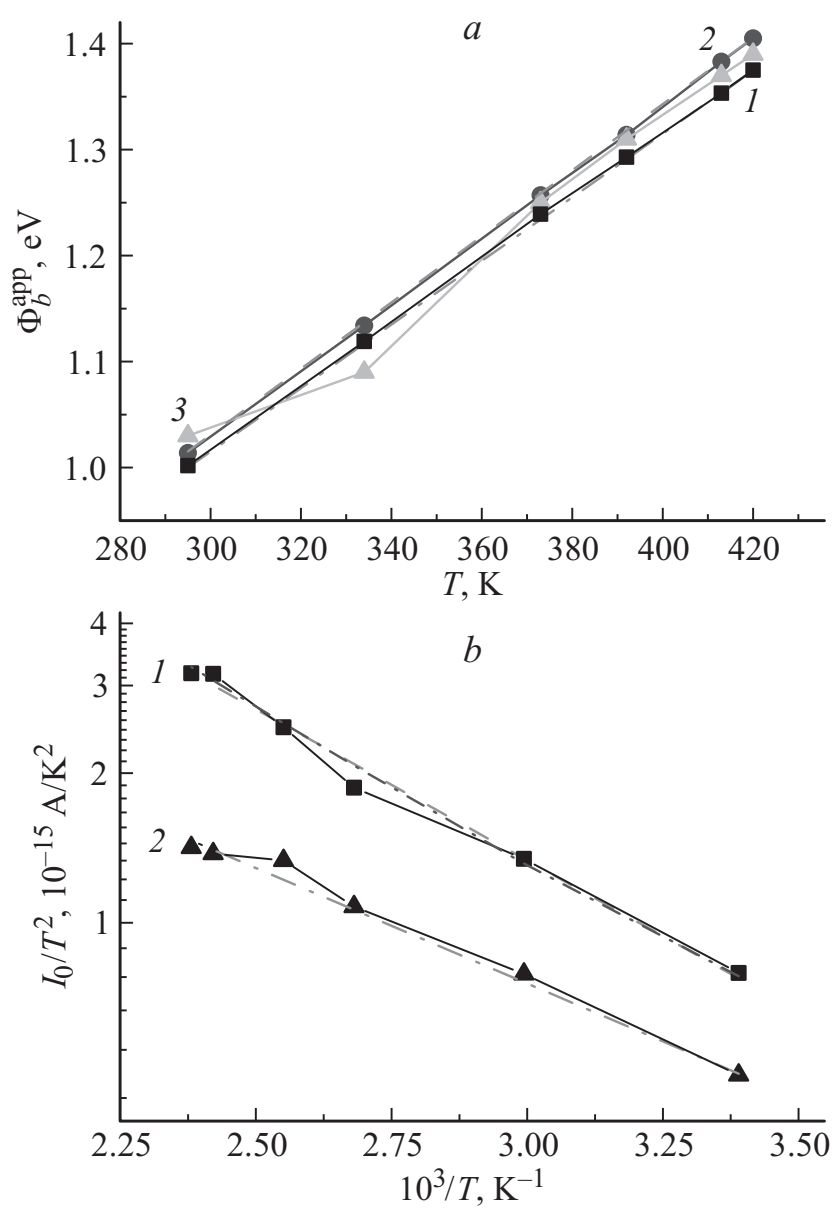

Рис. 6. $а$ - зависимость велчины барьеров элемента $\mathrm{Al} / n c-\mathrm{Si} / \mathrm{ITO}$, находящегося в атмосферном воздухе, от температуры: - высота барьера между пленкой $n c$-Si и нижним электродом $\Phi_{b 1 \text { app }}$ (см. рис. 1), 2 - высота барьеpa между пленкой $n c-\mathrm{Si}$ и верхним (пористым) электродом $\Phi_{b 2 \text { aрp }}, 3$ - высота $\Phi_{b 2 \text { арр }}$, вычисленная из аппроксимации $H(I)=I^{*} R_{s}+I /(I+I / n) \Phi_{b 2}$ (пояснения см. в тексте). Пунктирной и штрихпунктирной линиями показаны линейные аппроксимации в соответствии с соотношением $\Phi_{b \text { app }}(T)=\Phi_{b}(0)+k T\left(\alpha \chi^{0.5} \delta\right) . b-$ график Ричардсона для токов насыщения: $I_{s 1}-1$ и $I_{s 2}-2$. Пунктирной и штрихпунктирной линиями показаны соответствующие экспоненциальные аппроксимации.

исследованиями [19], согласно которым оболочки наночастиц кремния представляют собой $\mathrm{SiO}_{x}(0 \leq x \leq 2)$ с толщинами $\sim 1$ нм. Наличие слоя $\mathrm{SiO}_{x}(0 \leq x \leq 2)$ на поверхности наночастиц $\mathrm{Si}$ объясняет то, что величина барьеров Шоттки $\Phi_{b}^{\text {app }}$ при всех температурах образца превышает 1 эВ, другими словами, транспортные свойства структуры $\mathrm{Al} / n c$-Si film/ITO в большой степени определяются туннелированием носителей заряда через барьеры, образованные слоем $\mathrm{SiO}_{2}$.

Для того, чтобы определить эффективную постоянную Ричардсона, для изучаемого образца был построен график Ричардсона $\ln \left(I_{0} / T^{2}\right)$ в зависимости от $10^{3} / T$, причем в качестве $I_{0}(T)$ использовались значения $I_{01}$ и $I_{02}$ полученные в результате подгонки экспериментальных кривых по описанной выше методике.

График Ричардсона для токов насыщения $I_{01}$ и $I_{02}$ приведен на рис. $6, b$. На этом же рисунке приведены аппроксимации, соответствующие зависимостям

$$
\ln \left(\frac{I_{01,2}}{T^{2}}\right)=\ln \left(S A^{*}\right)-\delta \chi^{0.5}-\frac{\Phi_{b 1,2}(0)}{k T} .
$$

Величины барьеров $\Phi_{b 1}(0)$ и $\Phi_{b 2}(0)$, найденные из этих аппроксимаций, составляют 0.12 и 0.094 эВ соответственно, что с хорошей точностью соответствует значениям, найденным из линейных аппроксимаций $\Phi_{b}^{\mathrm{app}}(T)$ на рис. $6, a$.

Из зависимости (11), как это хорошо известно, можно найти величину эффективной постоянной Ричардсона, поскольку экстраполированное пересечение этой зависимости с осью абсцисс соответствует точке $\ln \left(S A^{*}\right)-\delta \chi^{1 / 2}$. Как уже отмечалось, для барьеров $\Phi_{b 1}$ и $\Phi_{b 2}$ величины $\delta \chi^{1 / 2}$ составляют 34.78 и 36.0, поэтому из аппроксимаций графиков рис. $6, b$ находим $A^{*}$, которая для $\Phi_{b 1}$ равна 113 , что практически совпадает с величиной $110 \mathrm{~A} \cdot \mathrm{cm}^{-2} \mathrm{~K}^{-2}$ для $n-\mathrm{Si}$ [17]. Для $\Phi_{b 2} A^{*}=86$. Эти результаты позволяют утверждать, что термоэмиссионная модель для данных структур может использоваться с хорошей точностью.

\section{4. Вычисление последовательного сопротивления структур $\mathrm{Al} / \boldsymbol{n c}-\mathrm{Si}$ film/ITO}

Как мы уже отмечали, из графиков функции $d\left(V_{2}\right) / d\left(\ln \left(I_{\text {exp }}^{*}\right)\right)$ и $H$ в зависимости от силы тока, протекающего через элемент $\mathrm{Al} / n c$-Si film/ ITO, можно определить последовательное сопротивление $R_{s}$ этого

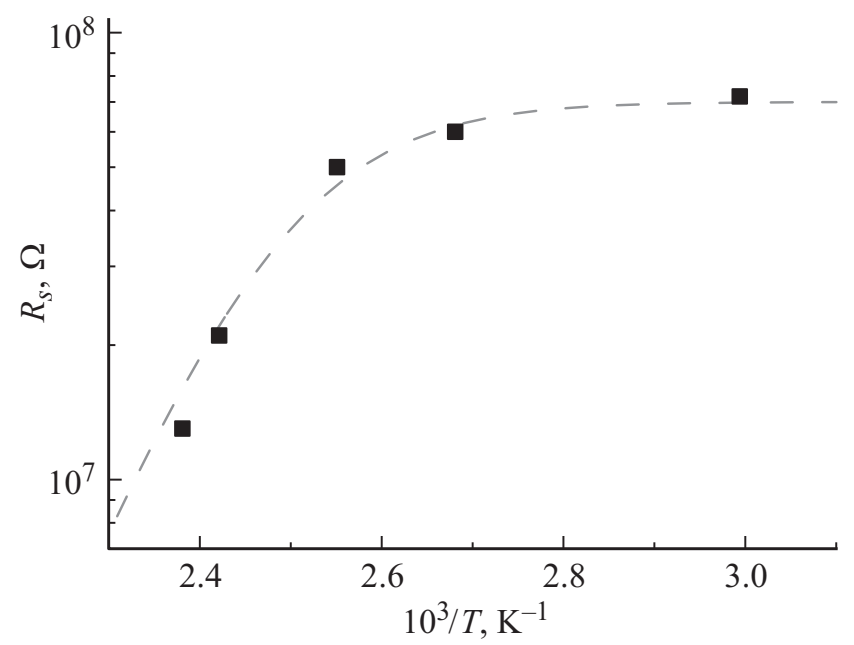

Рис. 7. Зависимость последовательного сопротивления $R_{s}$ элемента $\mathrm{Al} / n c$-Si/ITO, находящегося в атмосферном воздухе от обратной температуры. Пунктирными линиями показаны аппроксимации в соответствии с $R_{s}=r r_{0}$ $\times \exp \left(\frac{E}{k T}\right) /\left(r_{0} \exp \left(\frac{E}{k T}\right)+r\right)$. 
элемента. На рис. 7 приведена зависимость $R_{S}$ от обратной абсолютной температуры образца.

Из этого графика следует, что для зависимости $R s(1 / T)$ не подходит ни линейная, ни экспоненциальная функции. Аналогичный характер проводимости пленки из селеновых наночастиц наблюдался в работе [9], причем авторы публикации полагали, что хорошими аппроксимациями являются степенные функции $\sigma \sim T^{12}$ и $T^{20}$. Мы полагаем, что довольно трудно найти физическую причину, объясняющую такие большие степени в температурной зависимости.

Мы полагаем, что объяснить вид зависимости $R_{s}(1 / T)$ приведенной на рис. 7, можно, если предположить, что электрический транспорт в изучаемых образцах определяется одновременно двумя процессами с сильно различающимися энергиями активации. В предлагаемой упрощенной модели один процесс переноса зарядов является активационным с энергией активации $E$ $\left(r_{1}=r_{0} \exp (E / k T)\right)$, а второй безактивационный, причем оба процесса параллельно участвуют в транспортировке электрических зарядов. Соответственно суммарное сопротивление пленки $n c$-Si в исследуемых образцах должно определяться формулой

$$
R_{s}=\frac{r r_{0} \exp \left(\frac{E}{k T}\right)}{\left(r_{0} \exp \left(\frac{E}{k T}\right)+r\right)} .
$$

На рис. 7 приведена аппроксимирующая кривая, построенная в соответствии с этой моделью. Значения $E, r_{0}$ и $r$, определенные из этой аппроксимации, составляют 0.98 эВ, $4 \cdot 10^{-5}$ и $7 \cdot 10^{7}$ Ом.

\section{5. Импеданс спектроскопия структур $\mathrm{Al} / n c$-Si film/ITO}

Для того чтобы подтвердить или опровергнуть предположение об одновременной реализации в исследуемом образце двух видов проводимости, были проведены измерения импеданс-спектров структур в диапазоне температур $20-150^{\circ} \mathrm{C}$. На рис. 8 приведена зависимость мнимой компоненты $(\operatorname{Im} Z)$ импеданса образца $\mathrm{Al} / n c-\mathrm{Si} / \mathrm{ITO}$ от его действительной компоненты $(\operatorname{Re} Z)$ (Nyquist plot) для температур из указанного диапазона.

Из рисунка видно, что импеданс спектры имеют качественное отличие в диапазонах температур $20 \leq t \leq 50^{\circ} \mathrm{C}, 50<t \leq 150^{\circ} \mathrm{C}$. Для установления характера проводимости в изучаемых образцах были определены функции, которые могли с хорошей точностью аппроксимировать экспериментальные зависимости $Z=Z(\omega)$.

В первом температурном диапазоне вид экспериментальных зависимостей $\operatorname{Im} Z(\omega)$ и $\operatorname{Re} Z(\omega)$ может быть описан контуром, состоящим из параллельно соединенных импеданса Варбурга $\left(Z_{W}=A(1-j) / \sqrt{\omega}\right)[20]$, элемента постоянной фазы (Constant Phase Element - CPE) $\left(Z_{\mathrm{CPE}}=\frac{1}{C_{E}(j \omega)^{n}}, n<1\right)[21]$ и резистора $R$. В обеих формулах $\omega-$ угловая частота приложенного электриче-

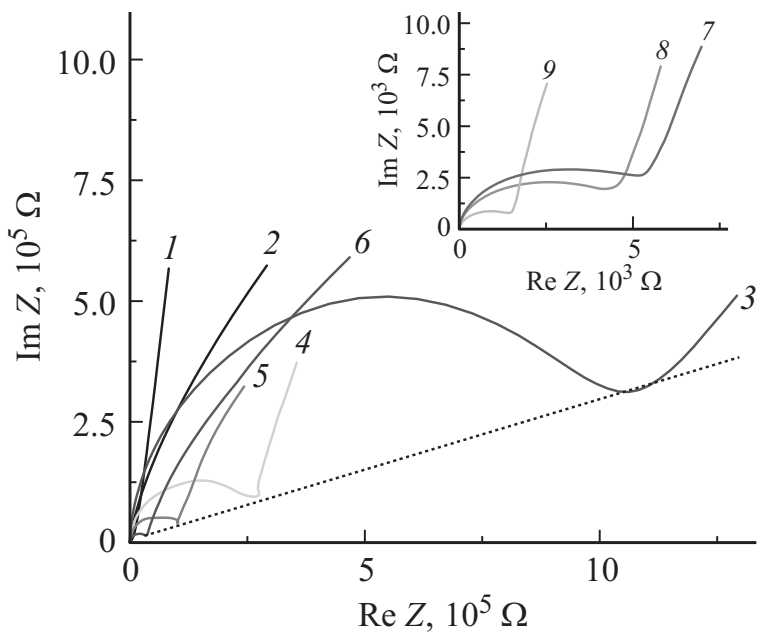

Рис. 8. Зависимость $\operatorname{In} Z(\operatorname{Re} z)$ импеданса элемента $\mathrm{Al} / n c-\mathrm{Si}$ film/ITO для различных температур. Температуры, при которых проводились измерения, ${ }^{\circ} \mathrm{C}: 1-22,2-42,3-55,4-61$, $5-72,6-104,7-114,8-136,9-146$. Зависимости для температур 114,136 и $146^{\circ} \mathrm{C}$ показаны на вставке рисунка.

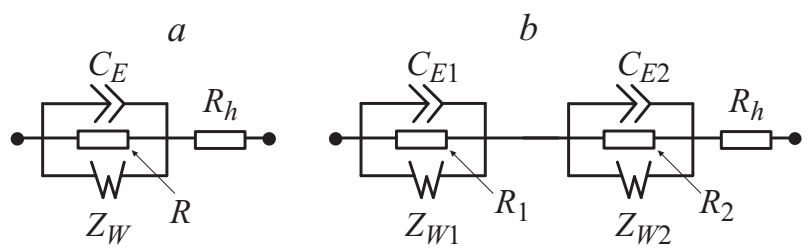

Рис. 9. Контуры, которые использовались для аппроксимации зависимостей $\operatorname{Im} Z(\omega)(\operatorname{Re} Z(\omega))$ : контур $1-$ при температурах, меньших $50^{\circ} \mathrm{C}(a)$, контур $2-$ при больших температурах $(b)$.

ского поля и $j=\sqrt{-1}$. Этот контур показан на рис. 9, $a$. На этом контуре также присутствует слабо зависящее от частоты высокочастотное сопротивление $R_{h}$.

Для аппроксимации зависимостей $\operatorname{Im} Z(\omega)$ и $\operatorname{Re} Z(\omega)$ при температурах образца, больших $50^{\circ} \mathrm{C}$, оказывается необходимым ввести еще один контур, последовательно соединенный с первым, который также состоит из параллельно соединенных импеданса Варбурга, СРЕ и резистора $R_{2}$ (см. рис. $9, b$ ).

Аппроксимация экспериментальных зависимостей $\operatorname{Im} Z(\operatorname{Re} Z)$ для образца при температурах 42 и $61^{\circ} \mathrm{C}$ показана на рис. 10, $a$ и $10, b$. На этих рисунках показана зависимость мнимой компоненты импеданса образца от его действительной компоненты (Nyquist plot).

Как известно, импеданс Варбурга указывает на существование диффузионно-контролируемых процессов переноса зарядов в электрохимических системах, СРЕ часто используется для того, чтобы описать частотную зависимость импеданса неидеальных конденсаторов, а контур СРЕ, параллельно соединенный с резистором, может применяться для описания электронной проводимости в неупорядоченных проводящих средах [22]. 

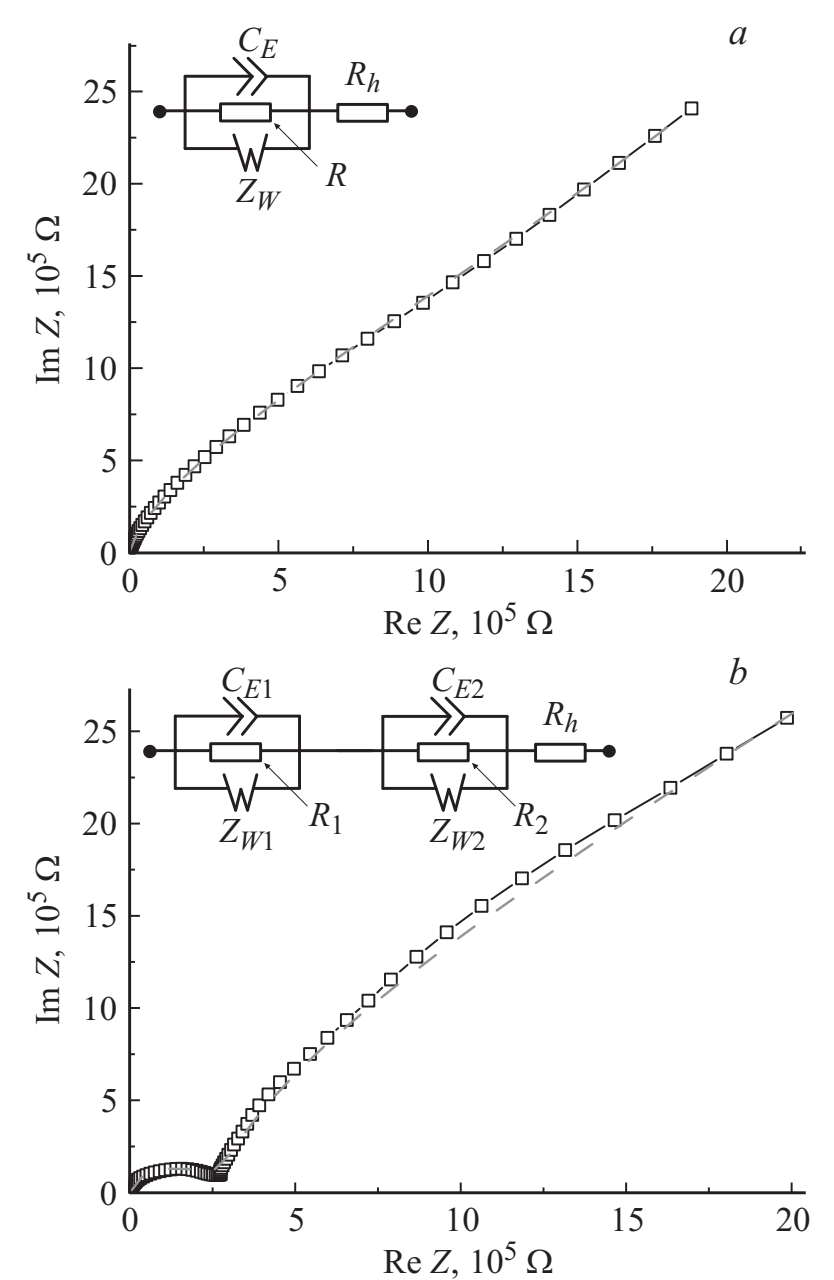

Рис. 10. Зависимость $\operatorname{Im} Z(\operatorname{Re} Z)$ импеданса элемента $\mathrm{Al} / n c-\mathrm{Si}$ film/ITO для температур, ${ }^{\circ} \mathrm{C}: a-42, b-61$. Пунктирными линиями показаны аппроксимации экспериментальных кривых с использованием контуров 1 и 2 соответственно.

Поэтому тот факт, что экспериментальная частотная зависимость импеданса образца может быть описана набором таких контуров, является доказательством того, что транспорт зарядов в изучаемых образцах определяется одновременно как ионной, так и электронной проводимостью. Анализ функций, аппроксимирующих экспериментальные кривые, позволяет определить соотношение $N=R / A$. Интуитивно ясно, что этот параметр связан с соотношением между диффузионной ионной и электронной проводимостью в образце и его увеличение свидетельствует о том, что в исследуемом образце доля ионной проводимости, связанной с диффузией, возрастает в общем процессе транспорта зарядов.

Так, функции, аппроксимирующей спектр рис. 10, $a$, соответствует значение $N=4$, а для аппроксимирующей функции рис. $10, b$ в области частот, меньших $10^{3}$ Гц, $N_{L}=2.2$ и в диапазоне больших частот $N_{H}=7.5 \cdot 10^{-3}$. Подобная динамика показывает, что, при наличии в образце обеих типов проводимости в области температур до $55^{\circ} \mathrm{C}$, преобладает диффузионная ионная проводи- мость. Однако, поскольку с ростом температуры образца уменьшается параметр $N$, уменьшается и доля ионной проводимости. Так, при температуре образца $\sim 60^{\circ} \mathrm{C}$ в области частот $\leq 10^{3}$ Гц вклад, который вносят электронная и ионная проводимости, сравним $\left(N_{L}=2.2\right)$, а в области высоких частот электронная проводимость становится доминирующим видом транспорта зарядов $\left(N_{H}=7.5 \cdot 10^{-3}\right)$. В диапазоне температур $60-140^{\circ} \mathrm{C}$ наблюдается подобное соотношение электронной и ионной проводимостей в исследованных структурах.

Наличие в исследованных образцах одновременно ионной и электронной проводимостей согласуется с описанием транспортных процессов в пористых пленочных электродах и наноструктурированных полупроводниках, контактирующих с электролитом, которое существует в современной научной литературе (см., например, [23,24]). Применительно к структурам, исследованным в этой работе, отметим наши более ранние результаты $[25,26]$, согласно которым ионная проводимость в $n c$-Si пленках определяется транспортом протонов вдоль поверхности наночастиц кремния. Источником протонов, участвующих в транспорте, являются молекулы воды, находящиеся в воздухе, окружающем пленки.

Таким образом, в данной статье мы сообщаем, что транспортные свойства пленок связаны одновременно как с протонной, так и с электронной проводимостью. Соответственно активационные зависимости изученных структур Al/nc-Si film/ITO (рис. 7) являются комбинацией двух процессов, связанных с ионной диффузей на поверхности наночастиц кремния и возбуждением электронов в зону проводимости этих наночастиц.

\section{4. Заключение}

В статье приводятся экспериментальные результаты по регистрации ВАХ тонкопленочных структур $\mathrm{Al} / n c-\mathrm{Si}$ film/ITO в температурном диапазоне $20-150^{\circ} \mathrm{C}$. Для двух диодов Шоттки, включенных навстречу друг другу, получена общая формула описывающая параметры этих диодов. На основе этой формулы построена вычислительная модель, обобщающая теоретические результаты работы [15] (S.K. Cheung и N.W. Cheung [15]), широко применяемые для анализа параметров одиночных диодов Шоттки. В результате нами разработана методика, позволяющая вычислять высоты барьеров Шоттки в системе двух диодов, включенных навстречу друг другу, коэффициенты их неидеальности и последовательные сопротивления таких структур.

Обнаружено, что в исследованном температурном интервале величины высот барьеров находятся вблизи значений $\sim 1$ эВ. Из анализа температурной зависимости высот барьеров установлено, что столь большие величины связаны с наличием на границах наночастиц кремния окисного слоя $\mathrm{SiO}_{x}(0 \leq x \leq 2)$, преодолевать 
который носители заряда могут как в результате теплового возбуждения, так и в результате туннелирования. Установлено, что собственные высоты барьеров Шоттки переходов $\mathrm{Al} / n c-\mathrm{Si}$ film и $n c-\mathrm{Si}$ film/ITO составляют $\sim 0.1$ эВ

Из анализа функций, аппроксимирующих экспериментальные импеданс-спектры структур $\mathrm{Al} / n c-\mathrm{Si}$ film/ITO, выяснено, что во всем исследованном диапазоне температур в структурах реализуется комбинированный механизм транспорта электрических зарядов, связанный с ионной (протонной) и электронной проводимостью. В диапазоне температур $\leq 55^{\circ} \mathrm{C}$ преобладающим видом проводимости является ионная проводимость. Однако с ростом температуры образца роль электронной проводимости в транспорте зарядов возрастает и в низкочастотном диапазоне $\left(v \leq 10^{3}\right.$ Гц) роли ионной и электронной проводимости сравниваются, а в высокочастотном диапазоне электронная проводимость становится доминирующей.

Работа выполнена при поддержке Российского фонда фундаментальных исследований (РФФИ), грант № 15-0209135.

\section{Приложение}

Вывод формулы (5) для $R=0$ :

$$
\begin{gathered}
I_{1}=I_{01} e^{\frac{e V_{1}}{k k T}}\left(1-e^{\frac{-e V_{1}}{k T}}\right) ; \\
I_{2}=-I_{02} e^{\frac{-e V_{2}}{n k T}}\left(1-e^{\frac{e V_{2}}{k T}}\right) ; \\
V_{1}+V_{2}=V ; \quad I_{1}=I_{2}=I .
\end{gathered}
$$

Введем обозначения:

$$
I_{01}^{\prime}=I_{01} e^{\frac{e V_{1}}{n k T}} e^{\frac{e e V_{1}}{k T}} ; \quad I_{02}^{\prime}=-I_{02} e^{\frac{-e V_{2}}{n k T}} e^{\frac{e V_{2}}{k T}},
$$

тогда формулы (П.1) и (П.2) примут вид:

$$
\begin{gathered}
I=I_{01}^{\prime}\left(e^{\frac{e V_{1}}{k T}}-1\right), \\
I=-I_{02}^{\prime}\left(e^{\frac{-e V_{2}}{k T}}-1\right) .
\end{gathered}
$$

Умножим (П.1') на $I_{02}^{\prime} e^{\frac{-e V}{2 k T}},\left(\Pi .2^{\prime}\right)$ на $I_{01}^{\prime} e^{\frac{e V}{2 k T}}$ и почленно сложим. В результате получим

$$
\begin{aligned}
I & =\frac{I_{01} I_{02} e^{\frac{e\left(V_{1}-V_{2}\right)}{n k T}} e^{\frac{-e\left(V_{1}-V_{2}\right)}{2 k T}}\left[e^{\frac{e V}{2 k T}}-e^{\frac{-e V}{2 k T}}\right]}{\left[I_{01} e^{\frac{e V_{1}}{k k T}}+I_{02} e^{\frac{-e V_{2}}{n k T}}\right]} \\
& =\frac{I_{01} I_{02}\left[e^{\frac{e V_{2}}{k T}}-e^{\frac{-e V_{1}}{k T}}\right]}{\left[I_{01} e^{\frac{e V 2}{n k T}}+I_{02} e^{\frac{-e V_{1}}{n k T}}\right]} .
\end{aligned}
$$

\section{Список литературы}

[1] R.T. Tang. Appl. Phys. Rev., 1, 011304 (2014).

[2] J. Yao, Zh. Sun, L. Zhong, D. Natelson, J.M. Tour. Nano Lett., 10, 4105 (2010).

[3] Y. Ye, B. Yu, Zh. Gao, H. Meng, H. Zhang, L. Dai, G. Qin. Nanotechnology, 23, 194004 (2012).

[4] E. Dubois, G. Larrieu. J. Appl. Phys., 96, 729 (2004).

[5] Sh.-Y. Chiu, H.-W. Huang, T.-H. Huang, K.-Ch. Liang, K.-P. Liu, J.-H. Tsai, W.-Sh. Lour. Sensors Actuators B, 138, 422 (2009).

[6] Jong-in Hahm, Ch.M. Lieber. Nano Lett., 4, 51 (2004).

[7] B. Yu, Y. Ye, P. Wu, Y. Dai, H. Zhang, L. Dai. Appl. Phys. Lett., 100, 143509 (2012).

[8] K. Tomioka, M. Yoshimura, T. Fukui. Nature, 488, 189 (2012).

[9] S. Sinha, S.K. Chatterjee, J. Ghosh, A.K. Meikap. J. Appl. Phys., 113, 123704 (2013).

[10] J. Huh, J. Na, J.S. Ha, S. Kim, G.T. Kim. ACS Appl. Mater. \& Interfaces, 3, 3097 (2011).

[11] X.-L. Tang, H.-W. Zhang, H. Su, Z.-Y. Zhong. Physica E, 31, 103 (2006)

[12] T. Nagano, M. Tsutsui, R. Nouchi, N. Kawasaki, Y. Ohta, Y. Kubozono, N. Takahashi, A. Fujiwara. J. Phys. Chem. C, 111, 7211 (2007).

[13] R. Nouchi. J. Appl. Phys., 116, 184505 (2014).

[14] L.S. Araujo, E.P. Bernardo, E.R. Leite. J. Phys.: Condens. Matter, 24, 225303 (2012).

[15] S.K. Cheung, N.W. Cheung. Appl. Phys. Lett., 49, 85 (1986).

[16] G.P. Kuz'min, M.E. Karasev, E.M. Khokhlov, N.N. Kononov, S.B. Korovin, V.G. Plotnichenko, S.N. Polyakov, V.I. Pustovoy, O.V. Tikhonevitch. Laser Phys., 10, 939 (2000).

[17] S. M. Sze, K.K. Ng. Physics of Semiconductor Devices, 3rd edn (John Wiley \& Sons, Inc., Hoboken, N.J., 2007) p. 137, ISBN-I 3: 978-0-47 1-1 4323-9.

[18] H.C. Card, E.H. Rhoderick. J. Phys. D: Appl. Phys., 4, 1589 (1971).

[19] С.Г. Дорофеев, Н.Н. Кононов, А.А. Ищенко, Р.Б. Васильев, М.А. Гольдштрах, К.В. Зайцева, В.В. Колташев, В.Г. Плотниченко, О.В. Тихоневич. ФТП, 43 (11), 1460 (2009). S.G. Dorofeev, N.N. Kononov, A.A. Ishchenko, R.B. Vasil'ev, M.A. Goldschtrakh, K.V. Zaitseva, V.V. Koltashev, V.G. Plotnichenko, O.V. Tikhonevich. Semiconductors, 43 (11), 1420 (2009).

[20] A. Lasia. Electrochemical Impedance Spectroscopy and its Applications. DOI 10.1007/978-1-4614-8933-7_1. (Springer Science + Business Media, N. Y., 2014), ISBN 978-1-46148933-7.

[21] J. Ross Macdonald. Sol. St. Ionics, 13, 147 (1984).

[22] J.C. Dyre, Th.B. Schrøder. Rev. Mod. Phys., 72 (3), 873 (2000).

[23] X. Ren, P.G. Pickup. J. Electroanalytical Chem., 420, 251 (1997).

[24] J. Bisquert, G. Garcia-Belmonte, F. Fabregat-Santiago, N.S. Ferriols, P. Bogdanoff, E.C. Pereira. J. Phys. Chem. B, 104, 2287 (2000).

[25] Н.Н. Кононов, С.Г. Дорофеев, Р.А. Миронов, В.Г. Плотниченко, Е.М. Дианов, А.А. Ищенко. ФТП, 54 (8), 1038 (2011). 
[26] N.N. Kononov, S.G. Dorofeev. Dielectric and Transport Properties of Thin Films Deposited from Sols with Silicon Nanoparticles. In. Smart Nanoparticles Technology, ed. by Abbass A. Hashim. ISBN 978-953-51-0500-8. Published by InTech, Janeza Trdine 9, 51000 Rijeka, Croatia, Chap. 19, 407.

Редактор А.Н. Смирнов

\title{
Characteristics of Schottky barriers of thin-film two-terminal Al/nano-silicon film/ITO structures
}

\author{
N.N. Kononov ${ }^{1}$, S.G. Dorofeev ${ }^{2}$ \\ ${ }^{1}$ Prokhorov General Physics Institute, \\ Russian Academy of Sciences, \\ 119991 Moscow, Russia \\ 2 Lomonosov Moscow State University, \\ Faculty of Chemistry, \\ 119991 Moscow, Russia
}

Abstract The temperature dependence of the Schottky barriers height and the series resistance of two-terminal thin-film structures $\mathrm{Al} /$ film of Si nanoparticles/ITO (Al/nc-Si film/ITO) was determined from the analysis of the current-voltage characteristics (CVC) in the temperature range $20-150^{\circ} \mathrm{C}$. It was found that the form of the CVC at all studied temperatures can be described by the model of two Schottky diodes incorporated toward each other (back-to-back Schottky diodes). The general formula is obtained for two back-to-back Schottky diodes, which allows to create a function approximating the experimental curves with high accuracy. On the basis of this formula is constructed computational model which generalizes the theoretical results of S.K. Cheung and N.W. Cheung is widely used for the analysis of curren-voltage characteristics of single Schottky diodes. As a result, we have developed a method which allows to calculate the height of the Schottky barriers in the system back-to-back Schottky diodes, the coefficients of their nonideality, and the series resistance of the system. It was found that the values of height of barriers are close to the values of $\sim 1 \mathrm{eV}$ in the investigated temperature range. It was determined, from the analysis of the temperature dependence of barrier heights, that such large values are related to the presence of the layer of oxide silicon $\mathrm{SiO}_{x}(0 \leq x \leq 2)$ on the boundaries of nanoparticles. It was found that the own heights of the Schottky barrier of junctions $\mathrm{Al} / n c$-Si film and $n c$-Si film/ITO close to $\sim 0.1 \mathrm{eV}$. From the analysis of the activation dependencies of series resistance of structures $\mathrm{Al} / n c$-Si film/ITO and from impedance spectra it was revealed that the transport of electrical charges is associated with the ion and electron conductivity. It was found that the contribution of the electronic conductivity in the overall process of transport of charges increases when the sample temperature increases. 